\title{
The calcium-phosphate balance, modulation of thyroid autoimmune processes and other adverse effects connected with thyroid arterial embolization
}

\author{
Grzegorz Kaminski • Andrzej Jaroszuk • \\ Ariadna Zybek $\cdot$ Krzysztof Brzozowski • \\ Piotr Piasecki $\cdot$ Piotr Ziecina $\cdot$ Marek Ruchala
}

Received: 29 July 2013/Accepted: 24 September 2013/Published online: 22 October 2013

(C) The Author(s) 2013. This article is published with open access at Springerlink.com

\begin{abstract}
In search of new treatment options for thyroid diseases, when conventional procedures are ineffective, contraindicated or associated with serious side effects, safety of thyroid arteries embolization in the treatment of particular thyroid diseases was evaluated. The study included eight subjects with retrosternal toxic goiter, six patients affected by Graves' disease, five cases of retrosternal non-toxic goiter, two subjects with post-amiodarone hyperthyroidism, and one patient with severe thyroidrelated orbitopathy, who underwent selective embolization of thyroid arteries. The study assessed and compared calcium-phosphate balance, modulation of thyroid autoimmunity and the presence of different side effects in patients who underwent the procedure. In addition, the serum concentrations of thyroid hormones, anti-thyroid autoantibodies and thyroid volume have been measured. Five of all enrolled subjects $(22.7 \%)$ experienced transient, not
\end{abstract}

G. Kaminski and A. Jaroszuk equally contributed to this work.

G. Kaminski · A. Jaroszuk

Department of Endocrinology and Isotope Therapy, Military

Institute of Medicine, Szaserów Street 128, 04-141 Warsaw,

Poland

e-mail: gkaminski@wim.mil.pl

\section{A. Zybek $(\varangle) \cdot$ M. Ruchala}

Department of Endocrinology, Metabolism and Internal

Medicine, Poznan University of Medical Sciences,

Przybyszewskiego Street 49, 60-355 Poznan, Poland

e-mail: ariadna.zybek@gmail.com

M. Ruchala

e-mail: mruchala@ump.edu.pl

K. Brzozowski · P. Piasecki · P. Ziecina

Department of Interventional Radiology, Military Institute of

Medicine, Szaserów Street 128, 04-141 Warsaw, Poland

e-mail: k.brzoza@wp.pl clinically relevant hypocalcaemia with no need for calcium supplementation. There were no significant changes in serum calcium levels in patients after embolization of both inferior thyroid arteries. The transient side effects associated with the treatment were neck pain and a slight increase in body temperature. Noted high concentration of free thyroid hormones immediately after the procedure was not accompanied by worsening of symptoms or signs of thyrotoxicosis. In patients with Graves' disease, a significant decrease in thyrotropin receptor antibodies level was observed. Thyroid arterial embolization does not disturb permanently calcium-phosphate balance, modulates positively thyroid autoimmune processes and is associated with no serious post-procedure side effects. Hence, it may be considered as a safe and effective treatment modality for selected thyroid disorders.

Keywords Thyroid embolization .

Thyroid diseases - Calcium-phosphate balance .

Thyroid autoimmunity $\cdot$ Side effects

\section{Introduction}

Thyroid gland diseases remain one of the most widespread problems in the modern clinical practice. It has been estimated that almost $9 \%$ of women and $2 \%$ of men are affected by a thyroid disorder [1]. Despite the existence of effective therapies for thyroid diseases, still some clinical cases require individual and alternative treatment modalities that would satisfy both patient and physician [2]. Standard therapeutic methods of hyperthyroidism include anti-thyroid drug therapy, radioiodine treatment with or without human TSH stimulation, and surgical procedures. Medical therapy may be connected with serious allergic 
Table 1 Characteristics of the patients enrolled in the study

\begin{tabular}{|c|c|c|c|c|c|c|}
\hline $\begin{array}{l}\text { Patient } \\
\text { no. }\end{array}$ & Age & Diagnosis/complication & $\begin{array}{l}\text { Pressure signs } \\
\text { yes/no }\end{array}$ & $\begin{array}{l}\text { Retrosternal } \\
\text { goiter yes/no }\end{array}$ & $\begin{array}{l}\text { Recurrence } \\
\text { yes/no }\end{array}$ & $\begin{array}{l}\text { Thyroid } \\
\text { volume }(\mathrm{ml})\end{array}$ \\
\hline 1 & 77 & Multinodular toxic goiter & Yes & Yes & Yes & 238 \\
\hline 2 & 60 & GD/unsuccessful ${ }^{131} \mathrm{I}$ treatment & No & No & No & 87 \\
\hline 3 & 54 & Post-amiodarone HT & No & No & No & 39 \\
\hline 4 & 73 & Multinodular toxic goiter & Yes & Yes & No & 269 \\
\hline 5 & 74 & Multinodular toxic goiter & Yes & Yes & No & 158 \\
\hline 6 & 44 & Multinodular toxic goiter & Yes & Yes & No & 265 \\
\hline 7 & 55 & Multinodular toxic goiter & Yes & Yes & No & 66 \\
\hline 8 & 72 & Post-amiodarone HT & No & No & No & 28 \\
\hline 9 & 52 & GD/resistant orbitopathy & No & No & No & 2,12 \\
\hline 10 & 52 & GD/unsuccessful ${ }^{131} \mathrm{I}$ treatment & No & No & No & 58 \\
\hline 11 & 66 & Multinodular toxic goiter & Yes & Yes & No & 315 \\
\hline 12 & 83 & Multinodular toxic goiter & Yes & Yes & No & 92 \\
\hline 13 & 31 & GD/anti-thyroid drugs intolerance & No & No & No & 110 \\
\hline 14 & 45 & Multinodular toxic goiter & Yes & No & No & 188 \\
\hline 15 & 47 & GD/unsuccessful ${ }^{131}$ I treatment & No & No & No & 87 \\
\hline 16 & 18 & GD/giant toxic goiter & Yes & Yes & No & 211 \\
\hline 17 & 51 & GD/giant toxic goiter & Yes & Yes & No & 263 \\
\hline 18 & 48 & Multinodular goiter & Yes & Yes & Yes & 91 \\
\hline 19 & 52 & Multinodular goiter & Yes & No & No & 166 \\
\hline 20 & 66 & Multinodular goiter & Yes & No & No & 83 \\
\hline 21 & 47 & Multinodular toxic goiter & Yes & Yes & No & 185 \\
\hline 22 & 64 & Multinodular goiter & Yes & No & Yes & 63 \\
\hline
\end{tabular}

$G D$ Graves' disease, $H T$ hyperthyroidism

reactions, agranulocytosis, thrombocytopenia, liver damage and many other adverse effects [3-7]. Anti-thyroid drugs intake during pregnancy sometimes results in congenital malformations and hypothyroidism of the fetus [3]. Effectiveness of the radioiodine intake may be easily reduced by earlier use of amiodarone, contrast agents, fast iodine metabolism or low iodine uptake in the thyroid [813]. In addition, there is a risk of orbitopathy development or deterioration during the radioiodine treatment $[14,15]$. The side effects associated with surgical procedures include post-operative hypoparathyroidism, recurrent laryngeal nerve damage and hemorrhage into the post-operative space [16-21].

The arterial embolization has been already widely used by interventional radiologists to treat vascular changes [22] as well as in oncology as a help in bleeding control and pain reduction or in various neoplasms management combined with locally administered chemotherapy [23, 24]. The efficacy of this technique in thyroid diseases was firstly evaluated by Galkin et al. [25], providing the evidence of thyroid volume reduction and prevention of goiter recurrence. Available data confirm usefulness of this method in patients affected by toxic goiter, recurrent goiter, Graves' disease (GD) or even thyroid carcinoma [2, 26-31]. The previous study conducted in our department supports these observations by showing that thyroid arterial embolization may be offered as an effective alternative for patients who will not or cannot accept standard therapy [32]. However, the influence of this procedure on calcium-phosphate balance and the autoimmune processes has not been fully evaluated to date.

The aim of our study was to assess the safety of thyroid arterial embolization in patients affected by various thyroid disorders.

\section{Subjects and methods}

\section{Subjects}

The studied group consisted of 22 consecutive patients (19 women and 3 men) aged from 18 to 83 years (mean $55.9 \pm 15.4$ years) referred to the department between 2004 and 2010, who met the inclusion criteria. The studied group included eight subjects with retrosternal toxic goiter, six patients affected by GD, five cases of retrosternal non-toxic goiter, two subjects with post-amiodarone hyperthyroidism, and one patient with severe thyroid-related orbitopathy. The 
enrolled group is characterized in Table 1. Thyrotoxicosis was in all affected patients caused by hyperthyroidism, and all cases of destructive thyrotoxicosis were excluded from the study.

Inclusion criteria were age over 18 years old, disqualification from surgical, radioiodine or medical therapy or lack of patient's consent to standard methods of treatment. Studied subjects were disqualified from surgical treatment because of old age, many accompanying cardiovascular and pulmonological entities or a huge, retrosternal goiter. In some cases of GD, no improvement occurred, despite a few attempts to radioiodine therapy. The post-amiodarone hyperthyroidism and multinodular goiter with only few autonomous regions are radioiodine resistant, due to low or limited thyroid parenchymal iodine uptake. Patients with anti-thyroid drug resistance or a poor medical therapy tolerance were also included into the study. One subject with active form of orbitopathy already treated with radioiodine and high doses of steroids took part in the study.

Diagnoses were based on medical histories, performed physical examinations, laboratory tests (thyroid hormones and anti-thyroid autoantibodies levels) and imaging techniques (ultrasound examination and thyroid scintiscan).

Before the embolization, all subjects underwent clinical examinations, blood tests and imaging studies. The serum concentrations of calcium $(\mathrm{Ca})$, phosphate $(\mathrm{P})$, anti-thyroid autoantibodies, including anti-thyroid peroxidase antibodies (AbTPO) and thyrotropin receptor antibodies (TRAb), thyroid stimulating hormone (TSH), free thyroxine (fT4), and free triiodothyronine (fT3) were measured. The size, volume and structure of the thyroid gland were pictured by computed tomography (CT) and ultrasonography (US). US-guided fine-needle aspiration biopsy of detected lesions was performed, if indicated.

The local bioethical committee approved the study, and all the participants gave written informed consent to participate.

\section{Arterial embolization}

Selective embolization of thyroid arteries was performed using the Seldinger technique. Before the procedure $1,000 \mathrm{ml}$ of isotonic saline solution and $1.0 \mathrm{~g}$ of methylprednisolone were infused to prevent possible adverse effects connected with contrast agents' infusion. The puncture site was chosen by the palpation of inguinal pulsation point in a patient placed in a supine position. After performing local anesthesia with $1 \%$ procaine, a small incision was made, through which, the angiographic catheter $(4 \mathrm{~F}-5 \mathrm{~F})$ was inserted into the left or right femoral artery. The movement of the catheter to the thyroid arteries was visualized by digital subtraction angiography. Before embolization, the selective angiography was performed to estimate the regions supplied with blood by each thyroid artery and the technical ability to occlude them. That provided important information, which helped to decide about the individual range of the procedure. The arteries to be embolized were chosen by the operator to achieve maximal reduction of the goiter size compromised with the practicability of the chosen procedure variant. To prevent hypoparathyroidism, no more than three arteries were occluded at a time. In most cases of diffuse goiter, three of main thyroid arteries were embolized; however, in patients with multinodular goiter, due to frequently impaired thyroid vascularization, sometimes only one pathological vessel needed to be occluded. Polyvinyl alcohol (PVA) or the mixture of histoacryl and lipiodol was injected directly to the blood vessels. In case of PVA, the procedure began with the infusion of smaller granules $(150-200 \mu \mathrm{m})$ to provide an effective occlusion without the risk of penetration of PVA into the systemic circulation. Subsequently, larger granules (200-300 $\mu \mathrm{m})$ were used to definitely block the lumen of the embolized vessel. The completeness of occlusion was checked by following angiography (Fig. 1). The procedure was performed under intravenous total anesthesia, due to a pain reaction resulting from acute ischemia of the thyroid gland. Afterwards, the catheter was removed and pressure dressing was applied. The embolization took approximately $2 \mathrm{~h}$. The patients were hospitalized and monitored for the next 7 days.

\section{Post-procedure management}

All patients received pain relieving treatment with nonsteroidal anti-inflammatory drugs or opioids, depending on the intensity of symptoms. Symptomatic treatment with beta-adrenergic blocking agents was introduced to prevent the occurrence of early thyrotoxicosis symptoms. The single infusion of methylprednisolone (250-500 mg) as well as treatment with prednisone $(30 \mathrm{mg} / 24 \mathrm{~h})$ for the following month was administered in all cases of GD.

Early post-procedure monitoring included $\mathrm{Ca}, \mathrm{P}, \mathrm{TSH}$, fT3, fT4 level evaluation at the 1st, 3rd and 7th day after embolization, and parathormon (PTH), if hypocalcemia had occurred. The latter follow-ups, showing distant postprocedure changes, were performed after 2 weeks, 1, 2, 3, 6,12 , and on average 20.3 months and consisted of clinical examinations, TSH, fT4, and fT3 measurements. Antithyroid autoantibodies serum concentrations were measured at baseline, after 3 months and at the end of the observation time. Depending on the results of the 1-month follow-up visit, the anti-thyroid drug therapy, beta-adrenergic blocking agents or thyroxin supplementation were introduced. Thyroid volume evaluation with CT was performed during 12-month follow-up visit. It has been assessed with standard formula of an ellipsoid with a 

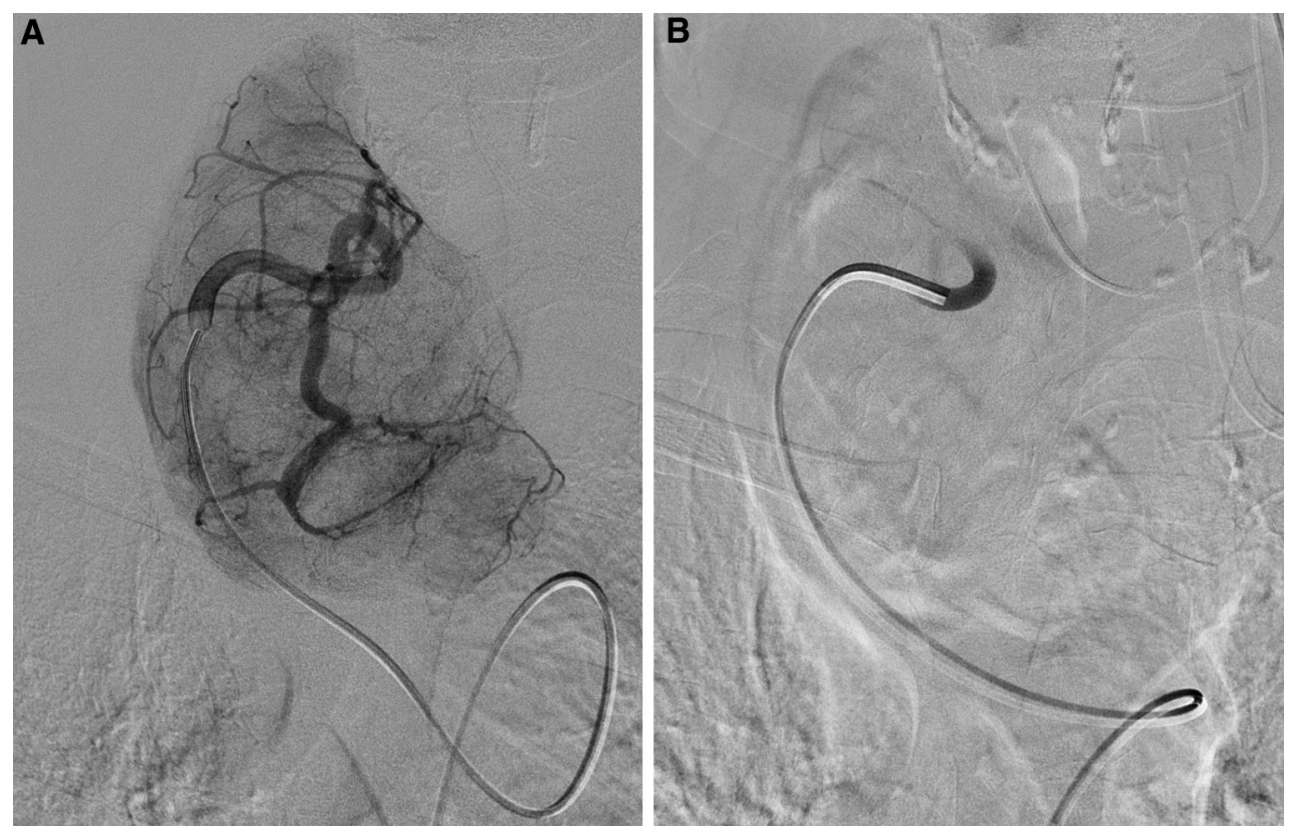

Fig. 1 Thyroid arterial embolization in patient affected by goiter development. a Selective angiography of the right inferior thyroid artery before the procedure. $\mathbf{b}$ Complete occlusion of the artery showed after the successful embolization

correction factor 0.5 (preferred range from 0.494 to 0.554 ) [33]. The average time of patients' post-procedure observation was $20.3 \pm 12.5$ months.

Serum assays and thyroid imaging

Anti-thyroid autoantibodies (AbTPO and TRAb) concentrations were assessed by radioimmunological method using AbTPO and TRAb RIA kits by BRAMHS. PTH level was measured with Liaison N-TACT test (normal range 17.3-72.9 pg/ml). Assessment of TSH, fT4 and fT3 serum concentration was performed using AutoDELFIA kit with a normal range of $0.55-3.55 \mu \mathrm{IU} / \mathrm{ml}, 7.64-19.7 \mathrm{pmol} / \mathrm{l}$, and 3.5-7.9 pmol/1, respectively. Ultrasound examination was performed using Hitachi EUB 8500 with a $7.5 \mathrm{MHz}$ frequency probe and computed tomography using GE LightSpeed 16.

\section{Statistical analysis}

Comparison of $\mathrm{Ca}$ and $\mathrm{P}$ serum concentrations in patients with two inferior thyroid arteries embolized and subjects with other variant of thyroid arterial embolization (two superior and one inferior thyroid arteries embolized, two superior thyroid arteries occluded, one superior and one inferior thyroid arteries embolized, and one pathological vessel occluded) was performed with the non-parametric Mann-Whitney test. The relevant parameters (TSH, fT4, fT3, AbTPO, TRAb serum concentration and thyroid volume) were compared with the use of a nonparametric
Table 2 The mean concentrations of calcium $(\mathrm{Ca})$ and phosphate (P) at baseline, 24 and $72 \mathrm{~h}$ after the procedure in the group of subjects with two inferior thyroid arteries embolized (2 i. t. a.) and in subjects with other variant of thyroid arterial embolization (two superior and one inferior thyroid arteries embolized, two superior thyroid arteries occluded, one superior and one inferior thyroid arteries embolized, or one pathological vessel occluded)

\begin{tabular}{|c|c|c|c|c|c|c|}
\hline \multirow[t]{2}{*}{ Parameter } & \multicolumn{2}{|l|}{ Baseline } & \multicolumn{2}{|l|}{$24 \mathrm{~h}$} & \multicolumn{2}{|l|}{$72 \mathrm{~h}$} \\
\hline & 2 i. t. a. & $\begin{array}{l}\text { Other } \\
\text { variant }\end{array}$ & 2 i. t. a. & $\begin{array}{l}\text { Other } \\
\text { variant }\end{array}$ & 2 i. t. a. & $\begin{array}{l}\text { Other } \\
\text { variant }\end{array}$ \\
\hline \multirow{2}{*}{$\begin{array}{l}\mathrm{Ca} \\
{[\quad[\mathrm{mmol} / \mathrm{l}]}\end{array}$} & 2.435 & 2.367 & 2.268 & 2.285 & 2.277 & 2.275 \\
\hline & \multicolumn{2}{|c|}{$p=0.251$} & \multicolumn{2}{|c|}{$p=0.780$} & \multicolumn{2}{|c|}{$p=0.977$} \\
\hline \multirow[t]{2}{*}{$\mathrm{P}[\mathrm{mmol} / \mathrm{l}]$} & 1.225 & 1.192 & 1.361 & 1.187 & 1.099 & 1.106 \\
\hline & \multicolumn{2}{|c|}{$p=0.731$} & \multicolumn{2}{|c|}{$p=0.017$} & \multicolumn{2}{|c|}{$p=0.951$} \\
\hline
\end{tabular}

Wilcoxon test. Statistical analysis was performed using data analyzing software system STATISTICA 7.1 (Stat Soft. Inc.).

\section{Results}

The difference in calcium level between the group of subjects with two inferior thyroid arteries embolized and subjects with other variant of thyroid arterial embolization 24 and $72 \mathrm{~h}$ after the procedure was not statistically significant. Five of all enrolled subjects (22.7 \%) experienced transient, not clinically relevant hypocalcaemia with no need for calcium supplementation. No significant decrease 
in average $\mathrm{Ca}$ level was observed 24 and $72 \mathrm{~h}$ after embolization. The phosphate level was significantly higher $24 \mathrm{~h}$ after the procedure in patients with two inferior thyroid arteries embolized (1.361 vs $1.187 \mathrm{mmol} / \mathrm{l} ; p=0.017$ ); however, $72 \mathrm{~h}$ after embolization no such difference was noted (1.099 vs $1.106 \mathrm{mmol} / \mathrm{l} ; p=0.951)$. All values are presented in Table 2.

The serum levels of anti-thyroid autoantibodies were significantly higher in subjects with GD (6 patients) than in group not affected by this disorder (16 patients). The average change in the serum level of AbTPO and TRAb was significantly higher in the group with GD than in the second group at 3-month follow-up and at the end of the observation time. However, in subjects with GD, only the decrease in TRAb level observed at the end of the follow-up was statistically significant (26 vs $6 \mathrm{U} / \mathrm{ml} ; p=0.043$ ). Significant changes in TRAb concentrations in subjects not affected by GD were noted neither at 3-month follow-up, nor at the end of the observation time. There was a significant increase in mean AbTPO level 3 months after the procedure, which eventually normalized after about 20.3 months (Table 3).

The early post-procedure side effects included: neck pain $(n=19 ; 86.4 \%)$, increased body temperature ( $n=12 ; 54.5 \%)$ and transient, not clinically relevant hypocalcaemia $(n=5 ; 22.7 \%)$.
The mean serum concentrations of TSH, fT4 and fT3 at baseline were $0.310 \mu \mathrm{lU} / \mathrm{ml}, 17.43 \mathrm{pmol} / \mathrm{l}$ and $8.505 \mathrm{pmol} / \mathrm{l}$, respectively. Statistically significant decrease in TSH serum level was observed $72 \mathrm{~h}$ and 7 days after the procedure, while during 3-month follow-up and at the end of the post-procedure observation time TSH level was within the reference range. Statistically significant increase in fT4 level was observed 24, $72 \mathrm{~h}, 7$ days and 2 weeks after embolization. Similarly, fT3 serum level significantly increased $72 \mathrm{~h}, 7$ days and 2 weeks after the procedure. After 2 months, the concentration of fT4 and fT3 normalized; however, fT3 level was significantly lower at the end of the follow-up than at the baseline. All the obtained values are presented in Table 4.

The state of euthyroidism or hypothyroidism has been reached in $70.6 \%$ of the studied group. However, this positive effect has been achieved in $81.8 \%$ of patients with multinodular goiter or post-amiodarone hyperthyroidism, and only in $50 \%$ of subjects with GD.

The thyroid volume in the studied group was significantly lower at 1-year follow-up than at baseline (139.3 vs $87.72 \mathrm{ml}, p=0.00004)$. The mean change in thyroid volume differs significantly, depending on a number of occluded arteries (1 artery, $62.0 \mathrm{ml} ; 2$ arteries, $25.6 \mathrm{ml} ; 3$ arteries, $78.8 \mathrm{ml} ; p=0.0401)$.

Table 3 Results of mean anti-thyroid autoantibodies (AbTPO, TRAb) serum levels assessment, at baseline and during follow-up, in patients who underwent thyroid arterial embolization

\begin{tabular}{|c|c|c|c|c|c|c|}
\hline \multirow[t]{2}{*}{ Parameter } & \multicolumn{3}{|c|}{ Patients with Graves' disease } & \multicolumn{3}{|c|}{ Patients without Graves' disease } \\
\hline & Baseline & 3 months & Av. 20.3 months & Baseline & 3 months & Av. 20.3 months \\
\hline \multirow[t]{2}{*}{ AbTPO [U/ml] } & 2,031 & 1,194 & 1,436 & 19.8 & 67.02 & 22.27 \\
\hline & & $p=0.513$ & $p=0.108$ & & $p=0.023$ & $p=0.066$ \\
\hline \multirow[t]{2}{*}{$\mathrm{TRAb}[\mathrm{U} / \mathrm{ml}]$} & 25.73 & 7.9 & 6.06 & 0.074 & 0.058 & 0.15 \\
\hline & & $p=0.109$ & $p=0.043$ & & $p=0.770$ & $p=0.503$ \\
\hline
\end{tabular}

Each $p$ value refers to the difference between the baseline value and that obtained at a particular time point. No statistical significance was reached between the values at different time points and is, therefore, not presented

Table 4 Mean serum concentrations of TSH [ $\mu \mathrm{IU} / \mathrm{ml}]$, fT4 (free thyroxin) [pmol/1] and fT3 (free triiodothyronine) [pmol/1] during follow-up, after thyroid arterial embolization compared with values before the procedure

\begin{tabular}{llllllllll}
\hline Parameter & Baseline & $24 \mathrm{~h}$ & $72 \mathrm{~h}$ & 1 week & 2 weeks & 1 month & 2 months & 3 months & Av. 20.3 months \\
\hline TSH [ $\mu \mathrm{IU} / \mathrm{ml}]$ & \multirow{2}{*}{0.310} & 0.284 & 0.034 & 0.016 & 0.221 & 1.229 & 0.406 & 0.759 & 1.308 \\
& & $p=0.767$ & $p=0.033$ & $p=0.008$ & $p=0.074$ & $p=0.209$ & $p=0.327$ & $p=0.027$ & $p=0.0029$ \\
fT4 [pmol/1] & 17.43 & 23.47 & 48.41 & 44.82 & 39.74 & 20.65 & 18.29 & 18.28 & 13.29 \\
& & $p=0.004$ & $p=0.0001$ & $p=0.0002$ & $p=0.0022$ & $p=0.509$ & $p=0.433$ & $p=0.664$ & $p=0.205$ \\
fT3 [pmol/1] & \multirow{2}{*}{8.505} & 7.714 & 17.520 & 19.38 & 14.87 & 8.146 & 7.715 & 8.283 & 5.803 \\
& & $p=0.125$ & $p=0.015$ & $p=0.003$ & $p=0.006$ & $p=0.108$ & $p=0.722$ & $p=0.723$ & $p=0.008$ \\
\hline
\end{tabular}

Each $p$ value refers to the difference between the baseline value and that obtained at a particular time point. No statistical significance was reached between the values at different time points and is, therefore, not presented 


\section{Discussion}

The arterial embolization is a widely described and broadly applied diagnostic and therapeutic method of modern invasive radiology. However, only few medical centers provided data about the possible advantages of arterial embolization in endocrinology [2, 25, 26, 29-32, 34].

To the best of our knowledge, full evaluation of calcium-phosphate metabolism in subjects who underwent thyroid arterial embolization has not been performed yet. As a prevention of potential parathyroid function disruption, it has been recommended not to embolize more than three main thyroid arteries and/or two inferior thyroid arteries at a time [2]. This theory was based on a fact that parathyroid glands are supplied with blood mainly by vessels originating from inferior arteries. In the current study, half of investigated cases $(n=12)$ had two inferior arteries occluded, but no significant difference in early $\mathrm{Ca}$ serum concentration was noted compared to other subjects. Approximately, $22.7 \%$ of the studied group experienced transient, not clinically relevant hypocalcaemia and no calcium supplementation was needed. Generally, the average serum calcium level in the studied group during the first few days after the procedure was within the normal range. Hence, in authors' opinion, there is no need to avoid simultaneous embolization of both inferior thyroid arteries; however, the obliteration of all four arteries may still be connected with a risk of hypoparathyroidism.

In accordance with the study by Zhao et al. [34, 35], patients with GD who underwent thyroid arterial embolization presented significant decrease in TRAb serum titer 6 months after the procedure. Results obtained in the present research seem to be in agreement with previous findings. Moreover, the serum concentration of aTPO also showed a downward trend, although it was not statistically significant. Described effect may be possibly connected with the decrease in thyroid parenchymal volume and lack of potential target for produced autoantibodies. On the other hand, in subjects without GD, a transient significant increase in aTPO serum concentration was shown that normalized at the end of the observation time. In authors' opinion, massive necrosis of the gland may be responsible for a self-limiting immunological response that did not persist; therefore, no constant autoimmune thyroid diseases were noted as a side effect of the performed procedure.

Xiao et al. [2] reported the normalization of the thyroid function in almost $64 \%$ of patients affected by GD (average time of observation: 6-50 months), while Zhao et al. [34] described the same positive effect in $71 \%$ of cases after 1 year and $59 \%$ after 3 years of observation. In the present study, $71 \%$ of hyperthyroid patients reached euthyroid or hypothyroid state $(80 \%$ affected by multinodular goiter and $50 \%$ by GD). The significant decrease in TRAb level and destruction of thyroid follicular cells surely play important roles in this process. Zhao et al. [30, 34] gave evidence that thyroid embolization has a significant influence on thyroid autoimmune processes, angiogenesis and apoptosis, which all together result in the remission of the disease. It is worth mentioning that patients who did not reach the euthyroid or hypothyroid state required lower doses of anti-thyroid drugs than before the therapy. The positive outcome in this group was associated with the lowering of adverse effects risk and better doctor-patient cooperation.

The evaluation of post-procedure side effects showed that most of them might have resulted from ischemia and following necrosis of the thyroid gland. The neck pain was most commonly observed with a frequency of $85 \%$, usually sufficiently treated only with non-steroidal antiinflammatory drugs. Only in two cases (9\%) opioids administration was necessary due to the high intensity of pain. Half of the patients experienced transient rise in body temperature that was not a result of an infection. No other serious complications occurred. These observations about the safety of the procedure were in accordance with those observed previously in our department and reported in the literature $[2,27-32,36]$.

In the previously published study by our team, we observed elevated values of fT4 and fT3 3 days after embolization, which showed quick reversal to near-normal or normal levels during follow-up [32]. Similarly, peak fT4 level was noted 3 days and fT3 level 7 days after embolization in present results, with a gradual normalization reached after 1 month. These observations are in agreement with these obtained by Xiao et al. [2], Zhao et al. [34, 37], and Dedecjus et al. [29]. This phenomenon may be explained by the destruction of thyroid parenchyma due to acute ischemia and necrosis of glandular tissue. Despite this transient postprocedure thyrotoxicosis, no symptoms of hyperthyroidism in euthyroid subjects appeared, as well as no symptoms worsening in patients already diagnosed as having hyperthyroidism. That may be partly attributed to prednisone and beta-adrenergic blocking agents administration.

The goiter volume reduction seems to be one of the most important aim in this kind of treatment. In previous studies, thyroid volume was assessed using CT scanning performed shortly after the therapy or based only on clinical examination $[2,29]$. Physical examination, as well as ultrasonography, could not provide precise estimation of thyroid volume, especially in case of retrosternal goiter. For that reason, CT performed before and after the arterial embolization (at the end of the observation time) seems to be the most applicable imaging technique to assess and compare the thyroid volume values. Generally, the mean change in thyroid volume was highly statistically significant in the present study $(51.58 \mathrm{ml}-37 \%)$ and the reduction in subjects with three 
obliterated arteries $(78.8 \mathrm{ml}-43.8 \%)$ corresponded with the clinical observation of Xiao et al. [2] Previously, CT examination performed 3 months after embolization visualized goiter volume reduction of approximately $32 \%$ of its original volume (from 13.0 to $76.3 \%$ ) with mean thyroid volume of $94 \mathrm{ml}$ [32]. Decrease in the size of goiter was accompanied with the compression symptoms improvement, especially in patients with a huge goiter.

The authors are aware that the enrolled group of patients is non-homogenous. The main criterion of inclusion was the inability to use the standard methods of treatment. Hence, thyroid arterial embolization seems to be an alternative or so-called last chance therapy and it should be performed on a large number of different disorders to prove its true applicability.

In conclusion, thyroid arterial embolization seems to be a worth considering novel treatment modality of thyroid disorders. This study suggests that this technique is rather safe, with no serious post-procedure side effects, no persistent calcium-phosphate balance disruptions, and short time of total anesthesia needed. Thyroid arterial embolization provided normalization of thyroid function in $70.6 \%$ of subjects and positive modulation of thyroid autoimmune processes. By the reduction of thyroid volume improvement of compression symptoms appears. However, due to small number of patients included and limited data from other medical centers, more prospective, randomized researches are needed to confirm these promising results.

Acknowledgments This study was funded from statutory sources of Department of Endocrinology and Isotope Therapy of Military Institute of Medicine in Warsaw.

Conflict of interest The authors declare that there is no conflict of interest that could be perceived as prejudicing the impartiality of the researches reported.

Open Access This article is distributed under the terms of the Creative Commons Attribution License which permits any use, distribution, and reproduction in any medium, provided the original author(s) and the source are credited.

\section{References}

1. T. Bjoro, J. Holmen, O. Kruger, K. Midthjell, K. Hunstad, T. Schreiner, L. Sandnes, H. Brochmann, Prevalence of thyroid disease, thyroid dysfunction and thyroid peroxidase antibodies in a large, unselected population. The Health Study of NordTrondelag (HUNT). Eur. J. Endocrinol. 143(5), 639-647 (2000)

2. H. Xiao, W. Zhuang, S. Wang, B. Yu, G. Chen, M. Zhou, N.C. Wong, Arterial embolization: a novel approach to thyroid ablative therapy for Graves' disease. J. Clin. Endocrinol. Metab. 87(8), 3583-3589 (2002)

3. D.S. Cooper, Antithyroid drugs. N. Engl. J. Med. 352(9), 905-917 (2005)

4. C.H. Lee, R.H. Liang, Antithyroid drug-induced agranulocytosis. Hong Kong Med. J. 5(4), 394-396 (1999)
5. M.H. Rosove, Agranulocytosis and antithyroid drugs. West. J. Med. 126(5), 339-343 (1977)

6. K. Takata, S. Kubota, S. Fukata, T. Kudo, E. Nishihara, M. Ito, N. Amino, A. Miyauchi, Methimazole-induced agranulocytosis in patients with Graves' disease is more frequent with an initial dose of $30 \mathrm{mg}$ daily than with $15 \mathrm{mg}$ daily. Thyroid 19(6), 559-563 (2009)

7. L. Gallelli, O. Staltari, C. Palleria, G. De Sarro, M. Ferraro, Hepatotoxicity induced by methimazole in a previously healthy patient. Curr. Drug Saf. 4(3), 204-206 (2009)

8. S.J. Bonnema, L. Bartalena, A.D. Toft, L. Hegedus, Controversies in radioiodine therapy: relation to ophthalmopathy, the possible radioprotective effect of antithyroid drugs, and use in large goitres. Eur. J. Endocrinol. 147(1), 1-11 (2002)

9. G.H. Daniels, Amiodarone-induced thyrotoxicosis. J. Clin. Endocrinol. Metab. 86(1), 3-8 (2001)

10. C. Rajeswaran, R.J. Shelton, S.G. Gilbey, Management of amiodarone-induced thyrotoxicosis. Swiss Med. Wkly. 133(43-44), 579-585 (2003)

11. A. Czarnywojtek, R. Czepczynski, M. Ruchala, R. Wasko, M. Zgorzalewicz-Stachowiak, E. Szczepanek, H. Zamyslowska, Z. Bartkowiak, E. Florek, J. Sowinski, Radioiodine therapy in patients with amiodarone-induced thyrotoxicosis (AIT). Neuro Endocrinol. Lett. 30(2), 209-214 (2009)

12. M. Ruchala, J. Sowinski, M. Dolata, R. Junik, M. Gembicki, A. Skiba, Radioiodine treatment of hyperthyroidism in patients with low thyroid uptake. Nucl. Med. Rev. Cent. East Eur. 8(1), 28-32 (2005)

13. S.J. Bonnema, S. Fast, L. Hegedus, Non-surgical approach to the benign nodular goiter: new opportunities by recombinant human TSH-stimulated 131I-therapy. Endocrine 40(3), 344-353 (2011)

14. A.W. Kung, C.C. Yau, A. Cheng, The incidence of ophthalmopathy after radioiodine therapy for Graves' disease: prognostic factors and the role of methimazole. J. Clin. Endocrinol. Metab. 79(2), 542-546 (1994)

15. S.H. Acharya, A. Avenell, S. Philip, J. Burr, J.S. Bevan, P. Abraham, Radioiodine therapy (RAI) for Graves' disease (GD) and the effect on ophthalmopathy: a systematic review. Clin. Endocrinol. (Oxf.) 69(6), 943-950 (2008)

16. A. Simsek Celik, H. Erdem, D. Guzey, F. Celebi, A. Celik, S. Birol, R. Kaplan, The factors related with postoperative complications in benign nodular thyroid surgery. Indian J. Surg. 73(1), 32-36 (2011)

17. E.I. Efremidou, M.S. Papageorgiou, N. Liratzopoulos, K.J. Manolas, The efficacy and safety of total thyroidectomy in the management of benign thyroid disease: a review of 932 cases. Can. J. Surg. 52(1), 39-44 (2009)

18. Y.S. Lee, K.H. Nam, W.Y. Chung, H.S. Chang, C.S. Park, Postoperative complications of thyroid cancer in a single center experience. J. Korean Med. Sci. 25(4), 541-545 (2010)

19. S. Kuy, S.A. Roman, R. Desai, J.A. Sosa, Outcomes following thyroid and parathyroid surgery in pregnant women. Arch. Surg. 144(5), 399-406 (2009). discussion 406

20. M.W. Seybt, S. Khichi, D.J. Terris, Geriatric thyroidectomy: safety of thyroid surgery in an aging population. Arch. Otolaryngol. Head Neck Surg. 135(10), 1041-1044 (2009)

21. J.C. Watkinson, Fifteen years' experience in thyroid surgery. Ann. R. Coll. Surg. Engl. 92(7), 541-547 (2010)

22. F. Perona, A. Barile, M. Oliveri, P. Quadri, C. Ferro, Superior thyroid artery lesion after US-guided chemical parathyroidectomy: angiographic diagnosis and treatment by embolization. Cardiovasc. Intervent. Radiol. 22(3), 249-250 (1999)

23. S. Bhansali, H. Wilner, J.R. Jacobs, Arterial embolization for control of bleeding in advanced head and neck carcinoma. J. Laryngol. Otol. 100(11), 1289-1293 (1986) 
24. M.M. De Vries, A.C. Persoon, P.L. Jager, J. Gravendeel, J.T. Plukker, W.J. Sluiter, T.P. Links, Embolization therapy of bone metastases from epithelial thyroid carcinoma: effect on symptoms and serum thyroglobulin. Thyroid 18(12), 1277-1284 (2008)

25. E.V. Galkin, B.S. Grakov, A.V. Protopopov, [First clinical experience of radio-endovascular functional thyroidectomy in the treatment of diffuse toxic goiter]. Vestn. Rentgenol. Radiol. (3), 29-35 (1994)

26. E.V. Galkin, [Interventional radiology in postoperative recurrent goiter]. Vestn. Rentgenol. Radiol. (6), 9-14 (1995)

27. H.E. Ramos, M. Braga-Basaria, C. Haquin, C.O. Mesa, L. Noronha, R. Sandrini, A. Carvalho Gde, H. Graf, Preoperative embolization of thyroid arteries in a patient with large multinodular goiter and papillary carcinoma. Thyroid 14(11), 967-970 (2004)

28. F. Tartaglia, F.M. Salvatori, D. Pichelli, M. Sgueglia, S. Blasi, F. Custureri, Preoperative embolization of thyroid arteries in a patient with a large cervicomediastinal hyperfunctioning goiter. Thyroid 17(8), 787-792 (2007)

29. M. Dedecjus, J. Tazbir, Z. Kaurzel, A. Lewinski, G. Strozyk, J. Brzezinski, Selective embolization of thyroid arteries as a preresective and palliative treatment of thyroid cancer. Endocr. Relat. Cancer 14(3), 847-852 (2007)

30. W. Zhao, B.L. Gao, M. Tian, G.F. Yi, H.Y. Yang, L.J. Shen, H. Li, J. Shen, Graves' disease treated with thyroid arterial embolization. Clin. Invest. Med. 32(2), E158-E165 (2009)
31. M. Dedecjus, J. Tazbir, Z. Kaurzel, G. Strozyk, A. Zygmunt, A. Lewinski, J. Brzezinski, Evaluation of selective embolization of thyroid arteries (SETA) as a preresective treatment in selected cases of toxic goitre. Thyroid Res 2(1), 7 (2009)

32. K. Brzozowski, P. Piasecki, P. Ziecina, E. Frankowska, A. Jaroszuk, G. Kaminski, R. Boguslawska-Walecka, Partial thyroid arterial embolization for the treatment of hyperthyroidism. Eur. J. Radiol. 81(6), 1192-1196 (2012)

33. W. Shabana, E. Peeters, M. De Maeseneer, Measuring thyroid gland volume: should we change the correction factor? AJR Am. J. Roentgenol. 186(1), 234-236 (2006)

34. W. Zhao, B.L. Gao, C.Z. Jin, G.F. Yi, H.Y. Yang, H. Li, D.P. Song, J.H. Hu, Y.N. Jiang, Long-term immunological study in Graves' disease treated with thyroid arterial embolization. J. Clin. Immunol. 28(5), 456-463 (2008)

35. W. Zhao, B.L. Gao, G.F. Yi, C.Z. Jin, H.Y. Yang, L.J. Shen, M. Tian, Y.Z. Yu, H. Li, D.P. Song, Apoptotic study in Graves disease treated with thyroid arterial embolization. Endocr. J. 56(2), 201-211 (2009)

36. W. Zhao, B.L. Gao, G.F. Yi, H.Y. Yang, H. Li, Thyroid arterial embolization for the treatment of hyperthyroidism in a patient with thyrotoxic crisis. Clin. Invest. Med. 32(1), E78-E83 (2009)

37. W. Zhao, B.L. Gao, H.Y. Yang, H. Li, D.P. Song, S.T. Xiang, J. Shen, Thyroid arterial embolization to treat Graves' disease. Acta Radiol. 48(2), 186-192 (2007) 